\title{
Présence de la Générale des Eaux au Mexique
}

\author{
The presence of Vivendi /Générale des Eaux in Mexico
}

\author{
par J. Couttelle \\ Vivendi/C.G.E. - OMSA
}

In Mexico, VIVENDI / Générale des Eaux is represented by OMSA (Operacion y Mantenimiento de Sistemas de Agua, S.A. de CV) a holding company created in 1989 by VIVENDI and ICA (Mexico's leading construction group). VIVIENDI / Générale des Eaux is present in Mexico through Omsa operational affiliates:

SAPSA (Servicios de Agua Potable, S.A.) for the contract of Mexico City, started on May 1993 for a 10 years period. OMSA AP (OMSA Agua Potable, S.A. de C.V.) the company responsible for potable water network Operation and Maintenance (O\&M) in the Mexico City contract. Created in July 1997, OMSA AP is also an actor in other cities such as Acapulco, where an O\&M contract for raw unto productions and pumping stations was signed after hurricane Paulina in October 1997. Guadalajara, with a sectorization one leak detection contract started for a pilot zone in this city.

CAASA (Concesionaria de Agua de Aguascalientes, S.A. de C.V.), responsible for potable water and sewage systems under a concession contract of 30 years, since October 1993.

SAASA (Servicios de Agua de Aguascalientes, S.A. de C.V.), operator of the Aguascalientes water system, owners of all the necessary assets for adequate managing of the contract by CAASA (work force and equipment).

OMSA PUEBLA, for the Puebla customer service contract, started in June 1998 for a duration of 10 years.

VIVENDI is leader in water systems management in Mexico, with over 820,000 subscribers and 6.5 millions of citizens served.

\section{I $\square$ PRÉSENTATION DES FILIALES DE VIVENDI/GÉNÉRALE DES EAUX AU MEXIQUE}

- SAPSA (Servicios de Agua Potable, SA de CV) pour le contrat de prestation de services de la ville de Mexico, entré en vigueur en mai 1993 pour une durée de 10 ans,

— OMSA A.P. (OMSA Agua Potable, SA de CV), société opératrice du contrat de Mexico pour les prestations techniques de maintenance de réseaux. Créée en juillet 1997, elle propose par ailleurs ses services à d'autres villes mexicaines. Acapulco : contrat de prestation de services pour la gestion de la production et des stations de reprise, suite au Cyclone "Paulina " d'octobre 1997. Guadalajara : contrat de sectorisation d'une zone pilote de la ville.

- CAASA (Concesionaria de Agua de Aguascalientes, SA de $\mathrm{CV}$ ), Concessionnaire du service d'eau potable et d'assainissement de la ville d'Aguascalientes depuis octobre 1993, pour une durée de 30 ans.

- SAASA (Servicios de Agua de Aguascalientes, SA de $\mathrm{CV}$ ), entreprise opératrice du service d'Aguascalientes qui détient la totalité des actifs nécessaires à l'exploitation de la concession. (Moyens humains, matériels et équipements).

- " OMSA PUEBLA", pour le contrat de prestation de ser- vices de la ville de Puebla, entré en vigueur en juin 1998 pour une durée de 10 ans.

Vivendi est leader de la distribution d'eau potable au Mexique, avec plus de 820000 abonnés et 6,5 millions d'habitants desservis.

\section{II $\square$ AGUASCALIENTES}

Ville située à $450 \mathrm{~km}$ au Nord-Ouest de Mexico, Aguascalientes (ancienne ville thermale) connaît une forte expansion démographique et économique $(3 / 4 \% /$ an $)$ : elle compte aujourd'hui de l'ordre de 800000 habitants et le niveau de vie est supérieur à la moyenne Mexicaine.

\subsection{Les caractéristiques du service}

Population : 800000 habitants

Nombre d'abonnés : 148000

Taux de croissance : environ $3 / 4 \%$ par an

Ressources en eau potable : aquifère profond - gestion de 174 forages répartis dans l'agglomération urbaine ( $95 \%$ de la population) et rurale - profondeur des forages comprise entre 300 et 600 mètres

Volume annuel produit : $87 \mathrm{Mm}^{3}$ (en 97)

Rendement théorique du réseau : estimé entre 45 et $50 \%$ 
(manque de moyens de comptage précis)

Caractéristiques physico-chimique de l'eau brute : température comprise entre $35^{\circ}$ et $45^{\circ}$, extraction importante de sable Chimiques de l'eau brute : dans $50 \%$ des forages (concentration : 4 à $6 \mathrm{~g} / \mathrm{m}^{3}$ ), taux de fluor compris entre 2 et $6 \mathrm{mg} / \mathrm{l}$ (norme : $1,5 \mathrm{mg} / \mathrm{l}$ )

Stérilisation : exclusivement au chlore gazeux et à l'eau de javel (couverture de $100 \%$ des forages)

Surexploitation de l'aquifère : le niveau dynamique de la nappe a baissé de 20 à 25 mètres entre les années 89 et 96 .

Linéaire du réseau d'eau potable : $1230 \mathrm{~km}$.

Zone sismique avec de nombreuses failles géologiques, dont l'origine est principalement due à la surexploitation de l'aquifère, qui traversent la ville en partie ou en totalité, ce qui génère des problèmes d'exploitation évidents (rupture des réseaux eau potable et assainissement, dégradation des forages, risques d'infiltration d'eau contaminée).

\subsection{Les dispositions principales du titre de concession}

La durée de la concession : 30 ans à compter d'octobre 93 .

La gestion complète du service. (Production, stérilisation, stockage, distribution et commercialisation).

Définition d'objectifs de résultats :

- Rendement technique du réseau : le rendement actuel du réseau est d'environ $50 \%$. L'objectif est d'atteindre $70 \%$ à partir de 2003 et jusqu'à l'échéance du contrat.

- Taux de recouvrement (montant encaissé/montant facturé) : le taux de recouvrement actuel est de $90 \%$. L'objectif contractuel est de maintenir un de recouvrement au minimun de $88 \%$.

Les moyens mis en œuvre pour atteindre ces résultats sont :

- la mise en place de campagnes de communication avec les usagers,

- l'amélioration du service,

- des mesures coercitives contre les mauvais payeurs.

Etablissement et révision des tarifs : nous disposons de cinq niveaux de tarif, trois attribués aux usagers domestiques établis en fonction des niveaux socio-économiques, et deux aux activités commerciales et industrielles. Par ailleurs, ces tarifs sont désormais actualisés mensuellement.

Mécanique d'application de la garantie : fiable et rapide en cas d'utilisation.

Création d'un organisme municipal régulateur :

La supervision est assurée par CCAPAMA, organisme décentralisé ayant pour mission de représenter la Municipalité dans ses relations avec la Concessionnaire. Il a un rôle de contrôle, de régulation et de surveillance des services d'eau potable et d'assainissement de la Concessionnaire. CCAPAMA dispose de plusieurs services spécialisés dans les activités opérationnelles, techniques, juridiques ou encore dans la gestion des abonnés. D'autre part, CCAPAMA doit veiller à la bonne application des règles d'attribution du Fond d'Aide Social (Fond de Apoyo Social), qui a pour objectif d'aider les familles les plus pauvres qui ne peuvent payer leur consommation d'eau au tarif minimun. (Sans aller jusqu'à la gratuité). Cet organisme dispose d'un Conseil d'Administration composé de représentants des trois niveaux de gouvernement (Municipal, Fédéral et Estatal), des deux partis politiques représentatifs (le PRI et le PAN), des entreprises locales et des citoyens d'Aguascalientes.

\subsection{Organisation de l'entreprise}

Avant la privatisation du service, le service des Eaux d'Aguascalientes était extrêmement centralisé : les 750 à 800 employés présents au moment de la privatisation du service étaient concentrés sur 2 sites et il n'y avait pas de division géographique du travail. L'organisation établie à partir de 1995 repose sur une division du territoire urbain d'Aguascalientes en quatre districts. La décentralisation de certaines activités commerciales et techniques s'est mise en place grâce, en particulier, au savoir-faire et à la sensibilité des agents Compagnie présents à Aguascalientes.

On distingue 3 niveaux organisationnels :

1/. Une gestion administrative centralisée

Elle regroupe la direction générale, un bureau d'études, le service informatique, la comptabilité, les ressources humaines, les achats, l'aire commerciale centrale et la communication sociale.

2/. Quatre districts (décentralisation de certaines activités commerciales et techniques).

Le service a été totalement réorganisé suite à sa concession dans le but de rationaliser l'exploitation, motiver les équipes et surtout améliorer la qualité du service offert aux usagers. La décentralisation de la gestion des abonnés, avec la création de 4 agences de proximité, est l'une des grandes améliorations organisationnelle.

Les districts sont placés sous la responsabilité d'un chef de district, ingénieur civil mexicain, et regroupent de 30 à 45000 abonnés. Chaque district a son autonomie et dispose :

- de son agence commerciale décentralisée en charge des relations administratives avec les abonnés (Contrat, Encaissement, Renseignements, Réclamations),

- de ses inspecteurs (4 à 5 par district). Ceux-ci ont les mêmes fonctions que nos fontainiers. Ils assurent avec l'aide de deux agents qui leur sont affectés toutes les tâches journalières de contrôle des forages et de leurs équipements, de manœuvres sur les réseaux et de l'entretien préventif des accessoires hydrauliques et des réservoirs,

- de ses équipes opérationnelles chargées au quotidien des réparations de fuites, des réparations des vannes, des renouvellements de branchements, des remplacements de compteurs...

- de deux électromécaniciens et d'un inspecteur assainissement qui établit les programmes préventifs d'entretien des réseaux d'assainissement.

En termes économiques, les résultats de cette réorganisation sont tout à fait significatifs. Les coûts d'exploitation sont en baisse de $20 \%$ par rapport à 1994. Pour la population, la proximité des agences est un progrès important dans la qualité du service. Cela s'inscrit dans une politique plus large qui consiste à personnaliser l'entreprise dans la ville. Cette politique englobe, par ailleurs, différentes campagnes de communication sociale qui ont pour objectif de créer une véritable culture de l'eau au travers d'actions dans les écoles, de journées d'information dans les quartiers populaires sur les économies d'eau et de campagnes d'information à travers les médias locaux.

\section{3/. Une aire technique centrale (Calpulli)}

Suite à la création des districts autonomes, les activités de l'aire centrale technique du "Calpulli " ont été réduites et concentrées sur un appui des districts pour les interventions lourdes : gestion des forages (système de vidéo, presse 
hydraulique), travaux principaux lourds sur réseaux d'eau potable et d'assainissement, contrôle de la qualité de l'eau (eau potable et assainissement), sectorisation et détection de fuites (trois véhicules équipés), maintenance du parc de véhicules (115 véhicules et 40 mobylettes), service de camions citernes (10 camions citernes, principalement pour les zones rurales) et maintenance des compteurs.

\section{- 2. 4. Transfert de Technologie:}

\subsubsection{Etude sur l'aquifere}

La situation gravissime de l'aquifère, l'abattement de son niveau statique de 4 à $5 \mathrm{~m}$ par an, les risques permanents de contamination par infiltration nous ont conduit avec la collaboration du BURGEAP et sur un financement FASEP à réaliser une étude intégrale sur le comportement de cet aquifère, sur l'établissement d'un modèle mathématique, sur l'élaboration de spécifications réglementaires sur l'extraction et l'usage de l'eau et enfin sur des spécifications techniques pour la réalisation de forages profonds.

\subsubsection{Formation des agents et amélioration de la qualité} des matériaux utilisés

Une formation a été assurée pendant plus d'une année par un technicien français sur la qualité des interventions et des contrôles de réparation ainsi que sur l'organisation du travail. Par ailleurs, des investigations ont été engagées pour déterminer les types de matériaux adaptés aux caractéristiques des installations existantes afin d'améliorer la qualité des interventions sur réseaux et branchements. Ces dispositions ont permis de réduire le nombre de fuites visibles, de plus de 20000 fuites en 95 à 13700 en 97 , soit une réduction de plus de $30 \%$ en deux ans.

\subsubsection{Macro et microcomptage}

Un travail d'analyse des conditions d'installation et de fonctionnement des compteurs en place, ainsi que des technologies de comptage micro et macro adaptées à la qualité et aux caractéristiques de l'eau, a été mené a bien. Cela a permis de mettre en place un programme de changements et d'installations de nouveaux compteurs. Ainsi, en 1997, 25000 nouveaux compteurs ont été installés, et il est prévu d'en installer 25000 autres en 1998. Fin 1997, plus de $90 \%$ des abonnés disposaient d'un compteur, l'objectif pour 1998 étant un taux de couverture supérieur à $95 \%$. Cette opération va augmenter de 16 à $18 \%$ le volume d'eau facturé.

\subsubsection{Sectorisation, détection des fuites non visibles}

Nous avons engagé un programme de sectorisation de l'ensemble du réseau d'eau potable de la ville d'Aguascalientes. La sectorisation vise les objectifs suivants :

- Réduction des pertes d'eau :

- détection des fuites non visibles,

- détection des branchements clandestins,

- rapidité d'intervention de réparation des fuites.

- Actualisation du fichier abonnés

- Améliorations du service :

- connaissance des anomalies locales,

- attention particulière aux problèmes rencontrés par les abonnés

- Gestion plus efficace :

- minimiser les gênes occasionées aux usagers,

- créer un outil d'opérateur pour suivre le comportement des réseaux,

- utilisation d'équipements et de matériels adaptés.
- Programmation pour le futur

- évolution des infrastructures d'eau potable à moyen et long terme,

- établir des programmes détaillés d'investissements productifs.

— En résumé, la méthodologie appliqué au Mexique permet :

- une réduction des coûts d'exploitation,

- une augmentation des recettes,

- une amélioration du service.

Pour atteindre ces objectifs, les différentes étapes de la sectorisation peuvent être décrites de la façon suivante :

- Etablissement des plans du réseau de distribution d'eau potable.

- Détermination en bureau d'études des zones sectorisées.

- Vérification sur le terrain des dites zones.

- Installation et renouvellement des vannes.

- Vérification de l'isolement des secteurs.

- Construction de regards de comptage.

- Analyse des flux et établissement de bilans hydrauliques nocturnes.

- Détection des fuites.

- Réparation des fuites.

- Validation et quantification des résultats obtenus.

Le programme de sectorisation a commencé à Aguascalientes en avril 1997 et doit s'achever au cours du deuxième semestre 1999 avec la création de l'ordre de 700 secteurs. L'objectif premier est d'améliorer le rendement du réseau en passant de $50 \%$ à $75 \%$. Nous avons aussi appliqué cette méthode dans d'autres villes au Mexique avec des résultats probants.

\section{MEXICO D.F.}

\subsection{Les caractéristiques du service}

Le District Fédéral se divise en 16 “ délégations ", dont 3, qui forment la zone centre-nord, ont été attribuées à notre filiale Sapsa : la délégation Cuauhtémoc, centre historique de la ville, la délégation Azcapotzalco, première zone industrielle du Mexique et la délégation Gustavo A. Madero, zone résidentielle populaire. Au total, 3,7 millions d'habitants et 420000 abonnés sont recensés dans la zone de Sapsa, zone très contrastée, en léger déclin démographique depuis le début des années 90 . Les actes délictuels y sont plus nombreux que dans le reste du DF.

Il existe trois autres opérateurs en charge de la ville de Mexico : Northwest Water/Gutsa, Tecsa (Lyonnaise des eaux/Bufete) et IASA (Brittingham/Servern Trent).

\subsection{Les dispositions contractuelles}

Le contrat est entré en vigueur en mai 93 pour une durée de 10 ans. Il se décompose en trois étapes qui ont, en règle générale, toutes démarré avec un retard de 1,5 à 2,5 ans pour des raisons économiques et politiques.

Première étape :

- Révision (réalisée à 100\%) du fichier abonnés (412 000 abonnés).

- Etablissement des plans des réseaux d'eau potable et d'assainissement : réalisation à $100 \%$ sur un système d'information géographique développé par la Compagnie Générale des Eaux. (Système Giris). 
- Installation de compteurs à lecture déportée : le pourcentage d'avancement est de $90 \%$ pour la la délégation Cuauhtémoc, $96 \%$ pour la délégation Azcapotzalco et $87 \%$ pour la délégation Gustavo A. Madero, ce qui représente un total de 282000 compteurs installés.

Deuxième étape :

- Actualisation permanente du fichier abonnés.

- Création de 6 agences ouvertes au public.

- Passage d'une facturation à prix forfaitaire à une facturation sur consommation réelle.

- Emission bimensuelle des factures : nous avons émis au 6ème bimestre 1997 la totalité des factures des abonnés, soit 412000 factures.

- Vérification du bon fonctionnement des compteurs et entretien préventif.

Troisième étape :

- Gestion des réseaux secondaires d'Eau Potable et d'Assainissement.

- Détection et réparation de fuites (sectorisation).

- Travaux de renouvellement et de ler établissement. (Branchements, vannes, raccordements...).

\subsection{Transfert de Technologie}

Le transfert de technologie et de savoir-faire s'est effectué tout au long du développement des trois étapes du contrat.

Lors du développement des activités de première étape, nous avons mis en place un système d'information géographique développé par la Compagnie Générale des Eaux, le système Giris, afin d'établir les plans des réseaux d'eau potable et d'assainissement et de disposer d'un outil de gestion.

Les principes de la sectorisation et de ses objectifs décrits ci-dessus ont été, de la même manière qu'à Aguascalientes, mis en œuvre à Mexico.

\section{IV $\square$ ACAPULCO}

\subsection{Les caractéristiques du service}

Population : 1,5 millions habitants

\section{- 4.2. Les dispositions contractuelles}

1998 : Contrat de prestation de services pour la gestion de la production d'eau, suite au Cyclone "Paulina " d'octobre 1997. Contrat signé sans appel d'offres compte tenu de l'ur- gence pour rétablir les infrastructures nécessaires aux services d'eau potable et d'assainissement.

\section{OMSA PUEBLA}

\section{Les caractéristiques du service}

Situation : $120 \mathrm{~km}$ à l'est de Mexico. Population : 2 millions habitants Nombre d'abonnés : 250000

\section{Les dispositions contractuelles}

1998 : Lancement d'un concours pour la modernisation du système commercial de l'organisme opérateur municipal de la ville. Le CA sur la durée du contrat s'élève à 45 millions de US\$. Il s'agit d'un contrat établi dans l'esprit de celui de la ville de Mexico mais sans la réalisation des plans de réseaux sur support informatique et sans les activités de gestion et de travaux de réseaux d'eau potable et d'assainissement. Il comporte les activités suivantes :

- Actualisation du fichier abonnés.

- Installation de 160000 compteurs.

- Implantation d'un logiciel informatique pour la gestion du service abonnés et l'établissement des factures.

- Ouverture de deux agences commerciales et réorganisation des six agences existantes. Gestion de l'ensemble des agences.

- Entretien du parc de compteurs.

Juin 1998 : Contrat gagné en juin 1998.

Août 1998 : Démarrage du contrat de Puebla.

\section{VI $\square$ CONCLUSION}

La délégation de gestion des services publics d'eau potable est naissante au Mexique, les rigidités administratives et politiques existantes sont en train progressivement de s'estomper et un dialogue de s'instaurer afin de permettre à l'industrie française des distributeurs d'eau et des équipementiers de s'installer progressivement et de participer, comme j'ai essayé de le décrire d'une manière pragmatique, à la résolution des problèmes multiples que peuvent connaître les 130 villes mexicaines de plus de 100000 habitants dans la gestion de leurs services publics d'eau potable. 